\title{
Molecular Classification of Gastric Adenocarcinoma
}

\author{
Qianqian Wang ${ }^{\mathrm{a}, \mathrm{b}}$, Ganglei Liu ${ }^{\mathrm{c}, \mathrm{d}}$, Chunhong $\mathrm{Hu}^{\mathrm{a}, \mathrm{d}}$
}

\begin{abstract}
As one of the leading causes of cancer-related deaths, gastric cancer (GC) has gained more and more attention. Although most GCs are adenocarcinomas, they have considerable heterogeneity among patients. Thus, appropriate classification and individualized treatment of GCs is essential. The traditional morphology-based classification systems including the World Health Organization (WHO) classification and the Lauren's classification have a limited utility in guiding clinical treatment due to the molecular heterogeneity of GC. Classifications based on molecular features become important. Recent years, molecular methods such as next-generation sequencing (NGS) including deoxyribonucleic acid (DNA) sequencing, ribonucleic acid (RNA) sequencing, whole-exome sequencing, copy number variation analysis and DNA methylation arrays have been used to classify the GC into molecular subtypes which can convey more detailed information of tumor than histopathological characteristics. In this review, we described the current molecular classifications of GC including the intrinsic subtypes, Lei subtypes, The Cancer Genome Atlas (TCGA) subtypes, Asian Cancer Research Group (ACRG) subtypes, and some other additional classifications.
\end{abstract}

Keywords: Gastric cancer; Molecular classification; Prognosis

\section{Introduction}

Most of the gastric cancers (GCs) are adenocarcinomas, which have considerable heterogeneity among patients [1]. The traditional morphology-based classification systems include the World Health Organization (WHO) classification (papillary,

Manuscript submitted April 22, 2019, accepted June 4, 2019

aDepartment of Oncology, The Second Xiangya Hospital, Central South University, Changsha, Hunan 410011, China

${ }^{b}$ Department of Oncology, The Affiliated Zhuzhou Hospital of Xiangya Medical College, Central South University, Zhuzhou, Hunan 412007, China

${ }^{\mathrm{c}}$ Department of Geriatrics Surgery, The Second Xiangya Hospital, Central South University, Changsha, Hunan 410011, China

${ }^{\mathrm{d} C o r r e s p o n d i n g ~ A u t h o r: ~ C h u n h o n g ~} \mathrm{Hu}$, Department of Oncology, The Second Xiangya Hospital, Central South University, 139 The Renmin Road, Changsha, Hunan 410011, China. Email: huchunh5829@126.com; Ganglei Liu, Department of Geriatric Surgery, The Second Xiangya Hospital, Central South University, 139 The Renmin Road, Changsha, Hunan 410011, China.

Email: liuganglei2005@126.com

doi: https://doi.org/10.14740/gr1187 tubular, mucinous, and poorly cohesive) [2], and the Lauren classification (intestinal, diffuse, and mixed) [3]. There is also an additional modified WHO classification (differentiated and undifferentiated) which is used to predict the risk of lymph node metastasis [4].

However, the accumulation of multiple genetic and epigenetic alterations which can lead to the dysregulation of oncogenes and tumor suppressors is considered the driver during the tumorigenesis [5]. The traditional morphology-based classification cannot convey the molecular heterogeneity of $\mathrm{GC}$ and cannot guide clinical practice in advanced GC for determining prognosis or predicting treatment responsiveness. Although the subclassification by molecular testing might increase the complexity of classification, identifying subtypes of $\mathrm{GC}$ based on molecular and genetic features is necessary to select targeted treatment [6].

This review aimed to update and summarize the molecular subtypes of gastric adenocarcinoma.

\section{Intrinsic Subtypes}

In 2011, Tan et al [7] identified new subtypes of GC by gene expression analysis of a large panel of GC cell lines, and tested their additional prognostic or predictive value in patients. They identified two major intrinsic subgroups of GC in 37 GC cell lines based on a genomic expression signature. These subtypes include genomic intestinal (G-INT) and genomic diffuse (GDIF) (Table 1) [7]. These subtypes were validated in primary tumors from 521 patients in four independent cohorts (Cohort 1 included 200 patients from the National Cancer Centre Singapore in Singapore; Cohort 2 included 70 patients from Peter MacCallum Cancer Centre in Australia; Cohort 3 included 65 patients from Yonsei University in South Korea; and Cohort 4 included 186 patients from the National Healthcare Group in Singapore).

These intrinsic subtypes were partially associated with Lauren's classification. The G-INT subtype was associated with intestinal histology, and the G-DIF subtype was associated with diffuse histology. However, the overall concordance between the intrinsic genomic subtypes and Lauren's classification was only $64 \%$ [7]. The two classifications are associated with highly distinctive gene expression patterns and biological pathways. They should be regarded as distinct. Genes upregulated in the G-INT subtype were related to carbohydrate and protein metabolism (fucosyltransferase 2 (FUT2)) and cell adhesion (lectin (LGALS4); cadherin 17, (CDH17)). The cell proliferation (aurora kinase B (AURKB)) and fatty acid metabolism (ELOVL family member 5 (ELOVL5)) functional 
Table 1. Intrinsic Subtypes

\begin{tabular}{|c|c|c|}
\hline & G-INT GC & G-DIF GC \\
\hline Histology & Intestinal histology (91/133) & Diffuse histology (64/107) \\
\hline $\begin{array}{l}\text { Molecular } \\
\text { alterations }\end{array}$ & $\begin{array}{l}\text { Genes up-regulated were related to carbohydrate and protein } \\
\text { metabolism (FUT2) and cell adhesion (LGALS4,CDH17) }\end{array}$ & $\begin{array}{l}\text { Cell proliferation }(A U R K B) \text { and fatty acid metabolism } \\
(E L O V L 5) \text { functional annotations were enriched }\end{array}$ \\
\hline \multirow[t]{2}{*}{ Treatment reaction } & $\begin{array}{l}\text { In vitro study, G-INT cell lines were } \\
\text { sensitive to } 5-\mathrm{FU} \text { and oxaliplatin }\end{array}$ & $\begin{array}{l}\text { In vitro study, G-DIF cell lines were more sensitive to } \\
\text { cisplatin }\end{array}$ \\
\hline & $\begin{array}{l}\text { Patients with G-INT tumors may derive benefit } \\
\text { from adjuvant 5-FU-based therapy }\end{array}$ & \\
\hline Prognosis & Superior overall survival & Poor \\
\hline
\end{tabular}

G-INT: genomic intestinal; G-DIF: genomic diffuse; GC: gastric cancer; AURKB: aurora kinase B; CDH17: cadherin 17; ELOVL5: ELOVL family member 5; FUT2: fucosyltransferase 2; LGALS4: lectin; 5-FU: 5-fluorouracil.

annotations were enriched in G-DIF subtype.

This study also observed a significant interaction between the intrinsic subtypes and benefit with chemoradiation [7]. In vitro study, G-INT cell lines were sensitive to 5-fluorouracil (5-FU) and oxaliplatin, while G-DIF cell lines were more sensitive to cisplatin. Furthermore, these intrinsic subtypes were also related to the clinical outcomes and turned to be prognostic factors. The G-DIF patients had poor prognosis compared with the G-INT patients in multiple patient cohorts, even after controlling for tumor stage [7]. On the other hand, the Lauren's classification did not show prognostic value. These results imply that clinically actionable information can be provided by genomic features. Intrinsic subtypes of GC might be used to determine prognosis and customize therapy.

\section{Lei Subtypes}

In 2013, Lei et al [1] provided another molecular classification of GC which was reproducible and biologically and therapeutically meaningful. After comparing gene expression patterns among 248 gastric tumors (Singaporean patients from the National Cancer Centre and hospitals of the National Healthcare Group, Australian patients from the Peter MacCallum Cancer
Centre in Melbourne), Lei et al [1] classified GCs into three independent subtypes: proliferative, metabolic, and mesenchymal (Table 2 [1]). These subgroups have different molecular and genetic features and different response to therapy.

The mesenchymal subtype over-represents the following gene sets: Kyoto Encyclopedia of Genes and Genomes (KEGG) focal adhesion, KEGG extracellular-matrix-receptor interaction, and Gene Ontology (GO) cell adhesion. The proliferative subtype was characterized by gene sets related to the cell cycle: KEGG cell cycle, KEGG deoxyribonucleic acid (DNA) replication, and $13 \mathrm{GO}$ gene sets. The metabolic subtype was characterized by gene sets from several KEGG metabolism pathways and GO digestion. The proliferative subtype was accompanied by high number of TP53 mutations while the rest two subtypes both showed a low number of TP53 mutations.

Mesenchymal-subtype gastric adenocarcinomas show cancer stem cell-like properties. The cell lines of this subtype are particularly sensitive to compounds targeting the phosphatidylinositol 3-kinase-AKT-mTOR (PI3K/Akt/mTOR) inhibitors in vitro study [8]. The metabolic subtype GCs were more sensitive to 5-FU treatment than the other subtypes. The proliferative-subtype patients had shorter disease-free survival than the other subtypes in multivariate analysis [1]. This classification of GC could guide development of therapies tailored

Table 2. Lei Subtypes

\begin{tabular}{|c|c|c|c|}
\hline & Proliferative GC & Metabolic GC & Mesenchymal GC \\
\hline Histology & Intestinal $(73.6 \%)$ & Intestinal $(53.6 \%)$ & Diffuse $(58.2 \%)$ \\
\hline \multirow[t]{2}{*}{$\begin{array}{l}\text { Molecular } \\
\text { alterations }\end{array}$} & $\begin{array}{l}\text { Characterized by gene sets related to } \\
\text { the cell cycle: KEGG cell cycle, KEGG } \\
\text { DNA replication, and } 13 \text { GO gene sets }\end{array}$ & $\begin{array}{l}\text { Characterized by gene sets from } \\
\text { several KEGG metabolism } \\
\text { pathways and GO digestion }\end{array}$ & $\begin{array}{l}\text { Over-represents the following gene sets: KEGG } \\
\text { focal adhesion, KEGG extracellular-matrix-- } \\
\text { receptor interaction, and GO cell adhesion }\end{array}$ \\
\hline & High number of TP53 mutations & Low TP53 mutations & Low TP53 mutations \\
\hline $\begin{array}{l}\text { Druggable } \\
\text { targets }\end{array}$ & & & $\begin{array}{l}\text { The PI3K-AKT-mTOR pathway } \\
\text { could be an effective drug target }\end{array}$ \\
\hline $\begin{array}{l}\text { Treatment } \\
\text { reaction }\end{array}$ & & $\begin{array}{l}\text { More sensitive to } \\
\text { 5-fluorouracil treatment }\end{array}$ & $\begin{array}{l}\text { The cell lines of this subtype were particularly } \\
\text { sensitive to compounds targeting the PI } 3 \mathrm{~K} / \\
\text { AKT/mTOR inhibitors in vitro study }\end{array}$ \\
\hline Prognosis & Shorter disease-free survival & & \\
\hline
\end{tabular}

GC: gastric cancer; KEGG: Kyoto Encyclopedia of Genes and Genomes; GO: gene ontology; PI3K/Akt/mTOR: phosphatidylinositol 3-kinase-AKTmTOR; DNA: deoxyribonucleic acid. 
Table 3. TCGA Subtypes

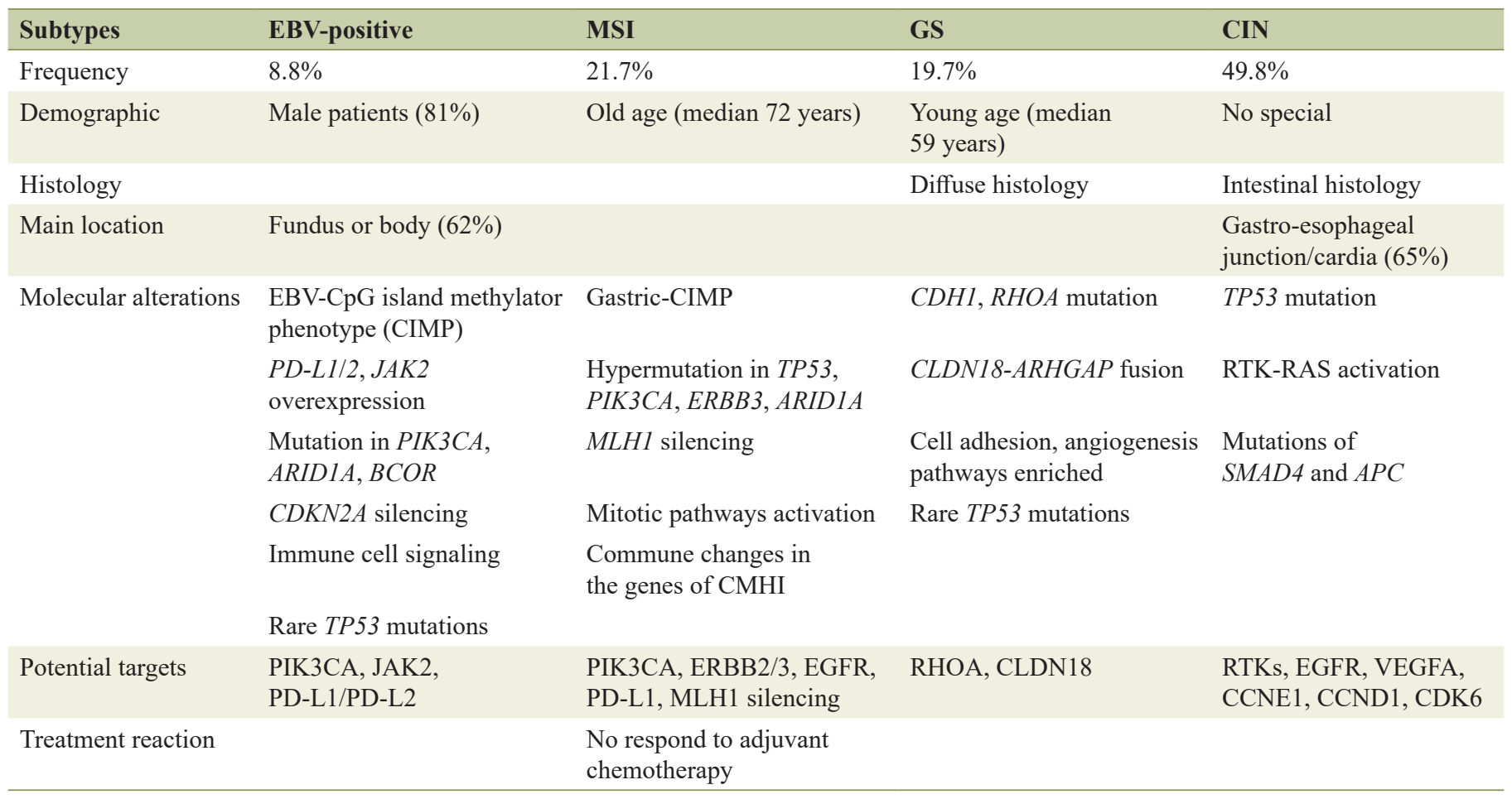

ARID1A: AT-rich interactive domain-containing protein 1A; BCOR: B-cell lymphoma 6 corepressor; CIN: chromosomal instability; GS: genomically stable; GC: gastric cancer; CIMP: CpG island methylator phenotype; DNMT3b: DNA methyltransferase 3b; EBV: Epstein-Barr virus; EGFR: epithelial growth factor receptor; ERBB2: Erb-B2 receptor tyrosine kinase 2; JAK2: Janus-associated kinase 2; LMP2A: latent membrane protein 2A; LELC: Iymphoepithelioma-like carcinoma; MSI: microsatellite instability; PI3K: phosphatidylinositol-3-kinase; RHOA: Ras homolog family member A; TCGA: The Cancer Genome Atlas; PD-L1/2: programmed death ligand-1/2; CDK6: cell division protein kinase 6.

to the molecular subtypes.

\section{The Cancer Genome Atlas (TCGA) Subtypes}

The most comprehensive molecular characterization of gastric adenocarcinoma was reported by TCGA Research Network in 2014 [9]. This study provided a roadmap for patient stratification and trials of targeted therapies. As part of TCGA project, 295 primary gastric adenocarcinomas were evaluated using six molecular platforms including array-based somatic copy number analysis, whole-exome sequencing, array-based DNA methylation profiling, messenger ribonucleic acid (RNA) sequencing, microRNA (miRNA) sequencing and reverse-phase protein array (RPPA). Microsatellite instability (MSI) testing was performed on all tumors. This study proposed a molecular classification dividing GC into four subtypes: tumors positive for Epstein-Barr virus (EBV) (8.8\% samples), microsatellite unstable tumors (21.7\% samples), genomically stable tumors (19.7\% samples), and tumors with chromosomal instability (49.8\% samples) (Table 3 [9]).

\section{EBV-positive GC}

This subtype of GC were mostly located in the gastric fundus or body $(62 \%)$, absent of intestinal metaplasia, and were more frequently found in male patients (81\%) [9]. This subtype was characterized by high EBV burden, extreme DNA hypermethylation, recurrent mutations in phosphatidylinositol 3-kinase (PIK3CA), AT-rich interactive domain-containing protein $1 \mathrm{~A}$ $(A R I D 1 A)$ and B-cell lymphoma 6 corepressor $(B C O R) \mathrm{mu}-$ tations, programmed death ligand-1/2 (PD-L1/2) overexpression, amplification of Janus-associated kinase 2 (JAK2), and Erb-B2 receptor tyrosine kinase 2 (ERBB2). This classification suggests potential therapeutic role for phosphatidylinositol-3-kinase (PI3K) inhibitors, JAK2 inhibitors and immune checkpoint antagonists.

This molecular subtype is due to infection by the EBV. Early entry of the virus into a single host cell leads to clonal expansion and cancer development $[10,11]$. In the latent state of infection, the virus is always in clonal episomal form [12].

Nine well-recognized viral genes (BARF0, BARF1, $B C L F 1, B H R F 1, B L L F 1, B R L F 1, B Z L F 1, E B N A 1$, and latent membrane protein $2 \mathrm{~A}(L M P 2 A))$ are highly expressed in EBVpositive GC [13-16]. Some of these genes have oncogenic potential [13]. Expression of $L M P 2 A$ is involved in up-regulation of survivin protein, which activates cellular DNA methyltransferase $3 b$ (DNMT3b), causing genome-wide aberrant methylation in host cells $[13,17]$. The typical genetic and epigenetic alterations define EBV-positive GC as a specific subtype of GC. 


\section{Microsatellite instability (MSI) GC}

This subtype was diagnosed at an older age (median age 72 years), with a slightly higher prevalence in female patients (56\%). This subtype is mostly due to promoter methylation which can lead to transcriptional silencing of the DNA mismatch repair gene $M L H 1$, resulting in a form of genomic instability known as microsatellite instability [18]. It showed elevated mutation rates, including mutations of genes encoding targetable oncogenic signaling proteins. Mutations in PIK3CA, epithelial growth factor receptor ( $E G F R$ ), ERBB2, and $E R B B 3$ were observed. The frequent frameshift mutations in repeat tracts of DNA cause inactivating mutations of key tumor suppressors, or frequent missense-activating mutations in oncogenes $[19,20]$. The MSI GC also had a high rate of PD-L1 expression.

MSI GCs have a predilection for antral location. They are associated with Helicobacter pylori (HP) infection, as well as intestinal metaplasia [19]. There is accompanying intense reactive lymphoid infiltration in both EBV and MSI subtypes. The EBV-positive type occasionally displays the lymphoepithelioma-like carcinoma (LELC) phenotype. Both molecular subtypes have been shown to display the $\mathrm{CpG}$ island methylator phenotype (CIMP) [16, 17, 21]. Also, both molecular subtypes are more commonly associated with intestinal histology.

\section{Genomically stable (GS) GC}

This subtype was signified by tumors with low somatic copynumber aberrations, and diagnosed more frequently in younger patients (median age 59 years). It was enriched for the diffuse histological variant. The GS tumors exhibited elevated expression of molecules in the cell adhesion and angiogenesis-related pathways, such as recurrent mutations of E-cadherin $(\mathrm{CDH} 1)$, Ras homolog family member $\mathrm{A}(\mathrm{RHOA})$. The $\mathrm{CDH} 1$ mutations underlie hereditary diffuse GC syndrome. The RHOA mutations or CLDN18-ARHGAP rearrangements may enhance invasiveness and disrupt intercellular cohesion and contribute to the diffuse histology found in $73 \%$ of this subtype.

\section{Chromosomal instability (CIN) GC}

The CIN GCs were found more frequently in the gastroesophageal junction/cardia (65\%), and exhibited an intestinal histology. It shows marked aneuploidy and focal activation of receptor tyrosine kinases-Ras (RTK/RAS) pathway. Amplification of ERBB2 (24\%), KRAS/NRAS (18\%), EGFR (10\%), ERBB3 (8\%), FGFR2 (8\%), and MET (8\%) were observed. This subtype also shows high frequency of TP53 mutations (73\%), amplification of genes encoding cell cycle mediators, such as cyclins E1, D1 (CCNE1, CCNDI) and cell division protein kinase 6 (CDKO). These findings are clinically important as they could be good therapeutic targets.

Several target drugs, trastuzumab (human epidermal growth factor receptor 2 (HER2) inhibitor), bevacizumab (vascular endothelial growth factor (VEGF) inhibitor) and ramucirumab (vascular endothelial growth factor receptor-2 (VEGFR2) inhibitor), were shown to be effective for treatment of GC. Other target drugs focusing on specific cancers with amplification of RTKs (e.g. cetuximab and panitumumab for EGFR, onartuzumab for MET, pertuzumab for HER2, and lapatinib for EGFR/HER2) could be effective anticancer strategies in GC [22-24]. There are also some other targeted agents including SMO inhibitors (sonidegib, buparlisib), PI3K inhibitors (BYL719, BKM120), AKT inhibitors (MK2206, GDC-0068), mTOR inhibitors (everolimus), and HGF/MET inhibitors (rilotumumab, onartuzumab, ABT-700, AMG337) [25].

Although the TCGA group was able to delineate four molecularly distinct subtypes of GC, no associated survival difference was observed within its cohort. Subsequently, retrospective studies reviewed that patients with the CIN subtype had the greatest survival benefit with adjuvant chemotherapy, whereas the GS subtype patients had the least associated benefit with adjuvant chemotherapy [26].

\section{Asian Cancer Research Group (ACRG) Sub- types}

In 2015 , by using the gene expression, genome-wide copy number microarray and targeted sequencing, the ACRG established clinically relevant molecular subtypes that could encompass the heterogeneity of $\mathrm{GC}$ and provide useful clinical information [27]. They analyzed the mRNA expression level of 300 tumors and classified them as MSI-high $(22.7 \%$ samples), microsatellite stable/epithelial-mesenchymal transition (MSS/EMT, 15.3\% samples), microsatellite stable/epithelial/ TP53 intact (MSS/TP53+, p53 active, 26.3\% samples), and microsatellite stable/epithelial/TP53 loss (MSS/TP53-, p53 inactive, $35.7 \%$ samples) [27, 28] (Table 4 [27]). Each molecular subtype is associated with distinct prognosis.

\section{MSI-high GC}

GCs of this subtype were found more frequently in the antrum (75\%), and exhibited an intestinal histology ( $>60 \%$ Lauren intestinal type). More than half of the cases were diagnosed at early stages. This subtype was associated with hypermutation in genes such as ARIDIA (44.2\%), the PI3K-PTEN-mTOR pathway (42\%), KRAS (23.3\%), and $A L K(16.3 \%)$. This subtype had the best prognosis and lowest frequency of recurrence $(22 \%)$ of the four subtypes. Liver-limited metastasis was dominant in $23 \%(6 / 26)$ of the MSI subtype and $21 \%(18 / 85)$ of the MSS/TP53- subtype, while the percentages in MSS/ EMT subtype and MSS/T53+ subtype were 4.6\% (3/64) and $8 \%(5 / 61)$. This further reinforced the clinical relevance of this classification.

\section{MSS/EMT GC}

GCs of this subtype were mostly diagnosed at a significantly 
Table 4. ACRG Subtypes

\begin{tabular}{|c|c|c|c|c|}
\hline Subtypes & MSI GC & MSS/EMT GC & MSS/TP53- GC & MSS/TP53+ GC \\
\hline Frequency & $22.7 \%$ & $15.3 \%$ & $35.7 \%$ & $26.3 \%$ \\
\hline Demographic & & $\begin{array}{l}\text { Diagnosed at a } \\
\text { significantly younger age }\end{array}$ & Male & Male \\
\hline Histology & Intestinal histology $(>60 \%)$ & Diffuse histology $(>80 \%)$ & Intestinal histology & Intestinal histology \\
\hline \multirow[t]{3}{*}{$\begin{array}{l}\text { Molecular } \\
\text { alterations }\end{array}$} & Silencing of $M L H 1$ gene & Loss of $C D H 1$ & $\begin{array}{l}\text { Highest prevalence of TP53 } \\
\text { and } R H O A \text { mutations }\end{array}$ & Frequent EBV infection \\
\hline & Overexpression of PD-L1 & & & \\
\hline & $\mathrm{T}$ cell infiltrate & & & \\
\hline Prognosis & $\begin{array}{l}\text { Best overall prognosis, lowest } \\
\text { recurrence rate }(22 \%)\end{array}$ & $\begin{array}{l}\text { Worst prognosis, highest } \\
\text { recurrence rate }\end{array}$ & Intermediate & Intermediate \\
\hline
\end{tabular}

ACRG: Asian Cancer Research Group; ARID1A: AT-rich interactive domain-containing protein 1A; CDH1: E-cadherin; GC: gastric cancer; MSS/EMT: microsatellite stable/epithelial-mesenchymal transition; MSS/TP53+: microsatellite stable/epithelial/TP53 intact; MSS/TP53-: microsatellite stable/ epithelial/TP53 loss; MSI: microsatellite instability; PI3K: phosphatidylinositol-3-kinase; RHOA: Ras homolog family member A; PD-L1: programmed death ligand-1.

younger age at advanced stages (III/IV), and exhibited diffusetype histology $(>80 \%)$. Tumor of this subtype had a lower number of mutation events. The MSS/EMT subtype had the worst prognosis and the highest recurrence frequency $(63 \%)$ compared to other subgroups. Peritoneal seeding was also frequently observed in this subtype (64.1\%) compared with others. This subtype also included a large set of signet ring cell carcinomas and showed loss of CDH1 expression.

\section{MSS/TP53+ GC}

This subtype has a higher prevalence of mutations in $A P C$, ARID1A, KRAS, PIK3CA, and SMAD4 compared with the MSS/TP53- subtype. EBV infection was more frequently observed in this subgroup compared to other groups. This subtype had the second best prognosis after MSI subtype.

\section{MSS/TP53- GC}

This subgroup had the highest rate of TP53 mutations (60\%) with a low frequency of other mutations. Recurrent focal amplifications in ERBB2, EGFR, CCNE1, CCND1, MDM2, ROBO2, GATA6, and MYC were commonly observed in tumors of this subtype.

EBV, MSI, CIN, and GS subtypes in TCGA correspond to the MSS/TP53+, MSI, MSS/TP53-, and MSS/EMT subtypes in ACRG. However, there were differences between the two classifications. The TCGA CIN and GS subtypes were observed across all ACRG subtypes. CDH1 mutations were more frequently observed in the GS subtype of TCGA $(37 \%)$ compared to the MSS/EMT subtype of ACRG (2.8\%). As one of main characteristics of the TCGA GS subtype, RHOA mutations were rarely detected in the ACRG MSS/EMT subtype, but were detected in the ACRG MSS/TP53- and MSS/TP53+ subtypes [29]. The main reasons for the partial overlap of these two classifications may be the different patient populations, tumor sampling, and technological platforms.

\section{Additional Subtypes Based on the TCGA and ACRG Classifications}

Setia et al [30] examined the expression of 14 biomarkers in a cohort of 146 gastric adenocarcinomas and performed unsupervised hierarchical clustering analysis using immunohistochemistry and in situ hybridization. They identified five groups of GC including EBV-positive GC (EBV GC, 5\% samples), microsatellite-instable GC (MSI-H GC, 16\% samples), and GC with aberrant E-cadherin expression (21\% samples), GC with aberrant p53 expression (51\% samples), and GC with normal p53 expression (7\% samples). The EBV GC showed a strong association with PD-L1. The MSI-H GC was associated with a lower frequency of nodal metastasis. The GC with aberrant E-cadherin subtype was associated with Lauren diffuse GC and had an aberrant pattern of E-cadherin staining. The GC with normal p53 had a MUC6 overexpression. The GC with aberrant p53 was associated with Lauren intestinal type GC and higher lymph node stage. The EBV and microsatellite-instable related adenocarcinomas showed a trend for superior survival. The five categories correspond to the TCGA and ACRG molecular subgroups. The authors offer a simplified and less expensive algorithm.

Ahn et al [31] classified a cohort of 349 successive gastric adenocarcinomas into five subtypes, on the basis of protein or mRNA expression of MLH1, E-cadherin, p53, and EBV. They found that these subtypes presented distinct clinicopathologic characteristics and corresponded to the ACRG and TCGA classifications. These five subtypes are EBV-positive tumors (EBV, 7.4\% samples), aberrant MLH1 expression (MSI, 6.9\% samples), aberrant E-cadherin expression (MSS/EMT, 15.2\% 
samples), aberrant p53 expression (MSS/p53+, 49.0\% samples), and normal p53 expression ((MSS/p53-, 21.5\% samples). The EBV tumors were mostly poorly differentiated type, following by molecular features: PIK3CA, ARID1A, $B C O R$ mutation, $C D K N 2 A$ promoter hypermethylation, $P D$ $L 1, P D-L 2$, and JAK2 amplification. The MSI tumors were mostly well and moderately differentiated type, accompanied by the following molecular features: MLH1 promoter hypermethylation, mutations in PIK3CA, ERBB2, ERBB3, $E G F R$, and $A R I D 1$. The EMT tumors were mostly diffuse type by Lauren's classification with $C D H 1, R H O A$ mutations. The MSS/P53+ tumors exhibited the molecular features including high TP53 mutation and HER2, EGFR, CCNE1, CCND1, MDM2, ROBO2, GATA6, and MYC amplification. The MSS/P53- tumors showed intermediate level of mutations $(A P C / K R A S / A R I D 1 / P I 3 K / S M A D 4)$. The EBV and MSI tumors showed better overall survival than the other types. The EMT tumors showed the poorest overall survival.

These studies all reproduced the previously reported molecular classification of GC using immunohistochemical analysis and in situ hybridization. In the foreseeable future, these methods may be used in clinical setting.

\section{Additional Subtypes and Classifications of GC}

The CIMP characterized by simultaneous methylation of the $\mathrm{CpG}$ islands of multiple genes, has been recognized as one of the most important mechanisms in gastrointestinal carcinogenesis [32]. Kusano et al [16] compared the clinicopathologic features of gastric carcinomas that had high CIMP methylation (CIMP-H) with tumors that had low CIMP methylation (CIMP-L) or negative CIMP methylation (CIMP-N). In their study, CIMP status was associated with distinct genetic, epigenetic, and clinicopathologic features in gastric carcinomas. Proximal location and less advanced pathologic TNM status contributed significantly to CIMP-H. Patients who had CIMP$\mathrm{N}$ GC had the worst survival. EBV-associated tumors were associated strongly with CIMP-H.

However, the existence of a comparable CIMP subtype in $\mathrm{GC}$ has not been clearly established. To further investigate this issue, Loh et al [33] performed comprehensive DNA methylation profiling of a well-characterized series of primary GC. The methylation status of 1,421 autosomal $\mathrm{CpG}$ sites located within 768 cancer-related genes was investigated. Unsupervised clustering of methylation data revealed the existence of six subgroups under two main clusters, referred to as L (low methylation; 28\% samples) and $\mathrm{H}$ (high methylation; $72 \%$ samples). The highly methylated group showed some features consistent with CIMP. No other significant differences in clinicopathological or molecular features were apparent, except that female patients were over-represented in the $\mathrm{H}$ tumor group.

As the complete spectrum of genetic changes in GC remains largely undefined, Wong et al [28] performed wholegenome sequencing of 49 GCs with diffuse $(n=31)$ and intestinal $(\mathrm{n}=18)$ histological subtypes and identified three mutational signatures, impacting TpT, $\mathrm{CpG}$ and TpCp (A/T) nucleotides. This study has provided an enhanced roadmap for the understanding of gastric adenocarcinoma with implications for future research.

\section{Conclusions}

To date, there is still a long way to go to classify GC into molecular subtypes perfectly. With the development of biotechnology, the molecular classification of GC will be more precise. The future GC treatment would be a clinical-pathological-molecular combined classification and guided individualized approach.

\section{Acknowledgments}

None to declare.

\section{Financial Disclosure}

None to declare.

\section{Conflict of Interest}

None to declare.

\section{Author Contributions}

Qianqian Wang contributed to drafting and revising the article. Chunhong $\mathrm{Hu}$ and Ganglei Liu contributed to revising the article.

\section{Abbreviations}

CIMP: $\mathrm{CpG}$ island methylator phenotype; GC: gastric cancer; GCA: gastric cardia adenocarcinoma; GNCA: gastric noncardia adenocarcinoma; G-INT: genomic intestinal; G-DIF: genomic diffuse

\section{References}

1. Lei Z, Tan IB, Das K, Deng N, Zouridis H, Pattison S, Chua $\mathrm{C}$, et al. Identification of molecular subtypes of gastric cancer with different responses to PI3-kinase inhibitors and 5-fluorouracil. Gastroenterology. 2013;145(3):554565.

2. Bosman FT, Carneiro F, Hruban RH, ND. WHO classification of tumours of the digestive system. International Agency for Research on Cancer; 2010.

3. Lauren P. The Two Histological Main Types of Gastric Carcinoma: Diffuse and So-Called Intestinal-Type Carcinoma. An Attempt at a Histo-Clinical Classification. Acta Pathol Microbiol Scand. 1965;64:31-49. 
4. Gotoda T. Endoscopic resection of early gastric cancer. Gastric Cancer. 2007;10(1):1-11.

5. Liu L, Cao L, Gong B, Yu J. Novel biomarkers for the identification and targeted therapy of gastric cancer. Expert Rev Gastroenterol Hepatol. 2015;9(9):1217-1226.

6. Bijlsma MF, Sadanandam A, Tan P, Vermeulen L. Molecular subtypes in cancers of the gastrointestinal tract. Nat Rev Gastroenterol Hepatol. 2017;14(6):333-342.

7. Tan IB, Ivanova T, Lim KH, Ong CW, Deng N, Lee J, Tan SH, et al. Intrinsic subtypes of gastric cancer, based on gene expression pattern, predict survival and respond differently to chemotherapy. Gastroenterology. 2011;141(2):476-485, 485 e471-411.

8. Visvader JE, Lindeman GJ. Cancer stem cells in solid tumours: accumulating evidence and unresolved questions. Nat Rev Cancer. 2008;8(10):755-768.

9. Cancer Genome Atlas Research N. Comprehensive molecular characterization of gastric adenocarcinoma. Nature. 2014;513(7517):202-209.

10. Yuen ST, Chung LP, Leung SY, Luk IS, Chan SY, Ho J. In situ detection of Epstein-Barr virus in gastric and colorectal adenocarcinomas. Am J Surg Pathol. 1994;18(11):1158-1163.

11. Shibata D, Weiss LM. Epstein-Barr virus-associated gastric adenocarcinoma. Am J Pathol. 1992;140(4):769-774.

12. Imai S, Koizumi S, Sugiura M, Tokunaga M, Uemura Y, Yamamoto N, Tanaka S, et al. Gastric carcinoma: monoclonal epithelial malignant cells expressing Epstein-Barr virus latent infection protein. Proc Natl Acad Sci U S A. 1994;91(19):9131-9135.

13. Zhao J, Liang Q, Cheung KF, Kang W, Lung RW, Tong $\mathrm{JH}$, To KF, et al. Genome-wide identification of EpsteinBarr virus-driven promoter methylation profiles of human genes in gastric cancer cells. Cancer. 2013;119(2):304312.

14. Sudo M, Chong JM, Sakuma K, Ushiku T, Uozaki H, Nagai H, Funata N, et al. Promoter hypermethylation of E-cadherin and its abnormal expression in EpsteinBarr virus-associated gastric carcinoma. Int J Cancer. 2004;109(2):194-199.

15. Kim J, Lee HS, Bae SI, Lee YM, Kim WH. Silencing and $\mathrm{CpG}$ island methylation of GSTP1 is rare in ordinary gastric carcinomas but common in Epstein-Barr virus-associated gastric carcinomas. Anticancer Res. 2005;25(6B):4013-4019.

16. Kusano M, Toyota M, Suzuki H, Akino K, Aoki F, Fujita M, Hosokawa M, et al. Genetic, epigenetic, and clinicopathologic features of gastric carcinomas with the $\mathrm{CpG}$ island methylator phenotype and an association with Epstein-Barr virus. Cancer. 2006;106(7):1467-1479.

17. Chang MS, Uozaki H, Chong JM, Ushiku T, Sakuma K, Ishikawa $\mathrm{S}$, Hino $\mathrm{R}$, et al. $\mathrm{CpG}$ island methylation status in gastric carcinoma with and without infection of Epstein-Barr virus. Clin Cancer Res. 2006;12(10):29953002.

18. Leung SY, Yuen ST, Chung LP, Chu KM, Chan AS, Ho JC. hMLH1 promoter methylation and lack of hMLH1 expression in sporadic gastric carcinomas with high-frequency microsatellite instability. Cancer Res.
1999;59(1):159-164.

19. Leung SY, Yuen ST, Chung LP, Chu KM, Wong MP, Branicki FJ, Ho JC. Microsatellite instability, EpsteinBarr virus, mutation of type II transforming growth factor beta receptor and BAX in gastric carcinomas in Hong Kong Chinese. Br J Cancer. 1999;79(3-4):582-588.

20. Myeroff LL, Parsons R, Kim SJ, Hedrick L, Cho KR, Orth K, Mathis M, et al. A transforming growth factor beta receptor type II gene mutation common in colon and gastric but rare in endometrial cancers with microsatellite instability. Cancer Res. 1995;55(23):5545-5547.

21. Toyota M, Ahuja N, Suzuki H, Itoh F, Ohe-Toyota M, Imai K, Baylin SB, et al. Aberrant methylation in gastric cancer associated with the $\mathrm{CpG}$ island methylator phenotype. Cancer Res. 1999;59(21):5438-5442.

22. Lordick F, Kang YK, Chung HC, Salman P, Oh SC, Bodoky G, Kurteva G, et al. Capecitabine and cisplatin with or without cetuximab for patients with previously untreated advanced gastric cancer (EXPAND): a randomised, open-label phase 3 trial. Lancet Oncol. 2013;14(6):490499.

23. Waddell T, Chau I, Cunningham D, Gonzalez D, Okines AF, Okines C, Wotherspoon A, et al. Epirubicin, oxaliplatin, and capecitabine with or without panitumumab for patients with previously untreated advanced oesophagogastric cancer (REAL3): a randomised, open-label phase 3 trial. Lancet Oncol. 2013;14(6):481-489.

24. Ohtsu A, Shah MA, Van Cutsem E, Rha SY, Sawaki A, Park SR, Lim HY, et al. Bevacizumab in combination with chemotherapy as first-line therapy in advanced gastric cancer: a randomized, double-blind, placebo-controlled phase III study. J Clin Oncol. 2011;29(30):3968-3976.

25. Strand MS, Lockhart AC, Fields RC. Genetics of gastric cancer. Surg Clin North Am. 2017;97(2):345-370.

26. Camargo MC, Kim WH, Chiaravalli AM, Kim KM, Corvalan $\mathrm{AH}$, Matsuo $\mathrm{K}$, Yu J, et al. Improved survival of gastric cancer with tumour Epstein-Barr virus positivity: an international pooled analysis. Gut. 2014;63(2):236243.

27. Cristescu R, Lee J, Nebozhyn M, Kim KM, Ting JC, Wong SS, Liu J, et al. Molecular analysis of gastric cancer identifies subtypes associated with distinct clinical outcomes. Nat Med. 2015;21(5):449-456.

28. Wong SS, Kim KM, Ting JC, Yu K, Fu J, Liu S, Cristescu $\mathrm{R}$, et al. Genomic landscape and genetic heterogeneity in gastric adenocarcinoma revealed by whole-genome sequencing. Nat Commun. 2014;5:5477.

29. Choi YY, Cheong JH. Beyond precision surgery: Molecularly motivated precision care for gastric cancer. Eur J Surg Oncol. 2017;43(5):856-864.

30. Setia N, Agoston AT, Han HS, Mullen JT, Duda DG, Clark JW, Deshpande V, et al. A protein and mRNA expression-based classification of gastric cancer. Mod Pathol. 2016;29(7):772-784.

31. Ahn S, Lee SJ, Kim Y, Kim A, Shin N, Choi KU, Lee CH, et al. High-throughput protein and mRNA expressionbased classification of gastric cancers can identify clinically distinct subtypes, concordant with recent molecular classifications. Am J Surg Pathol. 2017;41(1):106-115. 
32. Tahara T, Arisawa T. DNA methylation as a molecular biomarker in gastric cancer. Epigenomics. 2015;7(3):475486.

33. Loh M, Liem N, Vaithilingam A, Lim PL, Sapari NS,
Elahi E, Mok ZY, et al. DNA methylation subgroups and the $\mathrm{CpG}$ island methylator phenotype in gastric cancer: a comprehensive profiling approach. BMC Gastroenterol. 2014;14:55. 